\title{
Fatty Acid Synthesis in Rat Adipose Tissue
}

\author{
TRACER CONCENTRATION EFFECTS IN VITRO
}

\author{
By W. G. DUNCOMBE \\ The Wellcome Research Laboratories, Beckenham, Kent
}

(Received 28 July 1967)

\begin{abstract}
1. The use of labelled acetate for studying the synthesis of long-chain fatty acids in rat adipose tissue in vitro has been examined, with special reference to the effect of acetate concentration. 2. The incorporation of acetate into fatty acids is proportional to the concentration of acetate in the medium when the latter does not exceed about $10 \mu \mathrm{M}$. Above this concentration, the relative incorporation becomes progressively less, and reasons for this are discussed. 3. In particular it is shown that this is not necessarily due to disturbance of the endogenous rate of fatty acid synthesis by a relatively large amount of acetyl-CoA derived from added acetate. 4. However, to ensure that the added acetate does not cause such a disturbance its concentration must be kept sufficiently low. For labelled acetate used under present conditions, this concentration should not be more than about $10 \mu \mathrm{M}$.
\end{abstract}

The need to consider the chemical concentration of isotopic tracers to be used in biochemical experiments in vitro has been emphasized by Emerson \& Van Bruggen (1958). This is particularly important when a pool of low concentration is being labelled, for instance when acetate is being used to label acetyl-CoA. Defining a tracer concentration as one that will not influence the reaction being studied, Emerson \& Van Bruggen (1958) pointed out that the incorporation of the acetate label into a metabolite will, if such a concentration is exceeded, no longer quantitatively reflect the rate of reaction occurring before the introduction of the labelled acetate. That is, the tracer acetate will now behave as an exogenous substrate and not as a true tracer for the endogenous acetyl-CoA pool.

Work in this Laboratory on the metabolism of adipose tissue led to an investigation of the importance of this concentration effect when labelled acetate was being used as a tracer, for the biosynthesis of long-chain fatty acids in rat adipose tissue in vitro, the results of which are now reported. While these were being considered it became apparent that different interpretations of the results of Emerson \& Van Bruggen (1958) could be made according to the definition of the reaction being studied, and my results are discussed in the light of these considerations.

\section{EXPERIMENTAL}

Male Wistar rats (Chester Beatty strain) of 200-300 g. were killed by a blow on the head and the epididymal fat pads rapidly removed. For each experiment, comprising duplicate incubation vessels at a range of acetate concentrations from $0 \cdot 1 \mu \mathrm{M}$ to $10 \mathrm{mM}$, tissue from one rat was used. Each vessel contained about $50 \mathrm{mg}$. of tissue from each of the two contralateral fat pads, weighed on a torsion balance.

The incubation vessels were $20 \mathrm{ml}$. flat-bottomed screwcap vials, containing $2 \mathrm{ml}$. of Krebs-Ringer bicarbonate buffer (Krebs \& Henseleit, 1932) with appropriate substrates and tracers. After addition of tissue the vials were gassed with $\mathrm{O}_{2}+\mathrm{CO}_{2}(95: 5)$ for 2 min., capped and incubated for $3 \mathrm{hr}$. at $37^{\circ}$ in a metabolic shaking bath.

Sodium [1.14C]acetate (The Radiochemical Centre, Amersham, Bucks.) was used undiluted $(20-50 \mathrm{mc} / \mathrm{m}-\mathrm{mole})$ for the lower total concentration of labelled acetate. Higher concentrations were obtained by addition of appropriate amounts of unlabelled sodium acetate. Glucose (10 mM) was also added in some experiments.

After incubation the tissue was removed from the medium, washed briefly in water, blotted and sealed firmly in a screw-topped bijou bottle with $2 \mathrm{ml}$. of ethanolic $\mathrm{KOH}$ ( $2 \mathrm{ml}$. of aq. $40 \%, \mathrm{w} / \mathrm{v}, \mathrm{KOH}+10 \mathrm{ml}$. of ethanol). Saponification was carried out overnight in an oven at $100^{\circ}$. After addition of water to the hydrolysate, non-saponifiable lipids were extracted with light petroleum (b.p. 40-60 and discarded. The aqueous phase was acidified and extracted with light petroleum, and the extract was washed, dried over anhydrous $\mathrm{Na}_{2} \mathrm{SO}_{4}$ and evaporated to dryness on a steam bath. The molten fatty acids were allowed to cool in a vacuum desiccator and their radioactivity was determined by dissolving weighed amounts in a standard toluene-based scintillator and counting (at least 5000 counts) in a Packard Tri-Carb liquid-scintillation spectrometer. Counting efficiencies (about $85 \%$ ) were determined with internal standards, and appropriate corrections were made to the observed counting rates. Negligible radioactivity was found in fatty acids from tissue that had been inactivated by boiling before incubation. Radioactivity in media (before or after incubation) was determined by counting portions in a 
dioxan-based scintillator (Bray, 1960). Thin-layer chromatography of media after incubation (system of Braun \& Geenen, 1962) followed by scanning of the plates showed that about $95 \%$ of the radioactivity in the medium was in the form of acetate. Measurement of total radioactivity therefore gave a good indication of the amount of acetate not utilized during the incubation.

For each fatty acid sample the incorporation of labelled acetate, in moles/mg. of fatty acid, was calculated from the specific radioactivity of the fatty acids (disintegrations/ min./mg.) and that of the corresponding labelled acetate (disintegrations/min./mole).

\section{RESULTS AND DISCUSSION}

If labelled acetate is present in the incubation medium in such a concentration that it fulfils Emerson \& Van Bruggen's (1958) definition of a tracer, as already discussed, then labelling of the intracellular acetyl-CoA pool at a certain level will result and the fatty acids synthesized from this pool will, on merging with the existing tissue fatty acids, give rise to total fatty acids of a certain specific radioactivity. If the rate of synthesis of acetyl-CoA is proportional to the concentration of labelled acetate then an increase in the latter will result in the intracellular acetyl-CoA pool and the fatty acids synthesized from it having a correspondingly higher specific radioactivity, and we should expect the level of incorporation of labelled acetate into tissue fatty acids to be proportional to the extracellular labelled acetate concentration as long as the latter does not exceed a tracer level.

Fig. 1 shows the relationship between the concentration of labelled acetate and its incorporation into adipose-tissue fatty acids in three experiments, plotted on a log-log basis. [Emerson \& Van Bruggen (1958, plot as the ordinate the 'percent of anticipated response', a figure proportional to the acetate incorporation into fatty acids divided by the acetate concentration. A horizontal line in their results would thus become a straight line at $45^{\circ}$ when plotted on symmetrical log-log paper as in the present method.] It is seen that acetate incorporation into fatty acids is directly proportional to acetate concentration up to $10 \mu \mathrm{M}$, so that in this range the labelled acetate is undoubtedly behaving as a true tracer and not influencing in any way the course of the overall pathway: acetate $\rightarrow$ fatty acids. At concentrations greater than $10 \mu \mathrm{M}$ increasing deviations from proportionality occur, and the curves tend towards a plateau where increasing acetate concentration causes no further increase in acetate incorporation into fatty acids. This would be interpreted by Emerson \& Van Bruggen 1958 as showing that the increasing concentration of exogenous acetate is causing a progressive disturbance to the pathway.

Before the interpretation of the observed effects
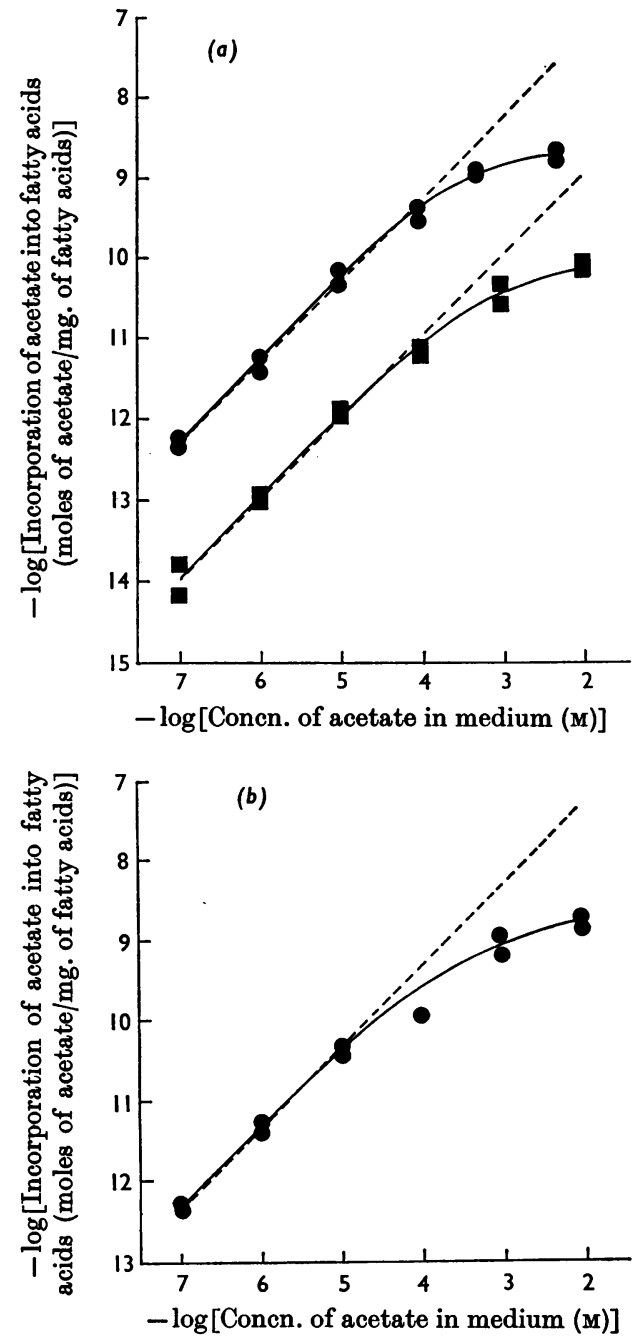

Fig. 1. Effect of concentration of labelled acetate in the medium on its incorporation into total fatty acids of rat adipose tissue in vitro. Each point represents one incubation vessel. Conditions were as described in the Experimental section. (a) $\bullet$, Medium with added glucose (10 mM); $\mathbf{\square}$, medium without glucose. (b) Another experiment with medium containing glucose (10 mM). Broken lines are the theoretical curves for exact proportionality between acetate incorporation and its concentration (drawn through point showing incorporation at acetate concentration of $1 \mu \mathrm{M}$ ).

is discussed it is necessary to define the reaction under investigation. If this is taken to be the sum of the steps leading from exogenous labelled acetate to labelled fatty acids, as is implied by Emerson \& Van Bruggen (1958) in their discussion then obviously their interpretation is valid. However, by definition the added labelled acetate is intended 
to act not as a substrate but as a tracer, and, since it is the metabolism of endogenous acetyl-CoA that is being traced rather than the incorporation into metabolites of exogenous acetate, it seems reasonable to consider that the reaction sequence being studied starts at the acetyl-CoA stage, though experimental results have of necessity to be expressed in terms of the added tracer. It may be pointed out here that Favarger (1965) has adversely criticized the use of any precursor other than glucose for the investigation of fatty acid synthesis, on the grounds of the uncertainty of the degree of penetration of acetate into tissues and of its activation to acetyl-CoA. These criticisms may be justified, but it should be noted that glucose itself is not necessarily the ideal tracer for this purpose. Thus ruminant mammary gland in vitro utilizes very much less glucose than acetate for fatty acid synthesis (Balmain, Folley \& Glascock, 1954), although glucose metabolism is active as shown by the degree of oxidation (Duncombe \& Glascock, 1956). Furthermore, experimental treatments might, by influencing the earlier stages of glucose catabolism, suggest a change in the rate of lipogenesis from labelled glucose without in fact having any effect on the enzymes of fatty acid synthesis. It seems therefore that, in spite of its disadvantages, acetate is a more generally valid tracer for acetyl-CoA.

Tracer conditions will therefore be defined as conditions in which the exogenous tracer acetate does not cause appreciable change in the concentration of endogenous acetyl-CoA. Taking this more limited definition it will be shown that the deviation from proportionality of the response curves of Fig. 1 at higher acetate concentrations does not necessarily imply that a tracer concentration has been exceeded.

The form of the response curves (Fig. 1) at low acetate concentration suggests that up to about $10 \mu \mathrm{M}$ the rate of formation of labelled acetyl-CoA from labelled acetate is proportional to the concentration of the latter (assuming that the endogenous pool of acetyl-CoA that is being labelled is of constant size), and that in this range the amount of labelled acetyl-CoA being produced does not appreciably increase the size of the endogenous acetyl-CoA pool. At acetate concentrations higher than $10 \mu \mathrm{M}$ the shape of the curves may be influenced by a number of factors.

First, the specific radioactivity of the total acetyl-CoA pool may not be increasing linearly with increasing labelled acetate concentration, owing to an inverse dilution effect. A curve of the type shown in Fig. 2 can be derived from a theoretical consideration of such an effect. It can be seen that as the amount of labelled acetyl-CoA derived from labelled acetate becomes significant compared with the size of the endogenous acetyl-CoA pool so does

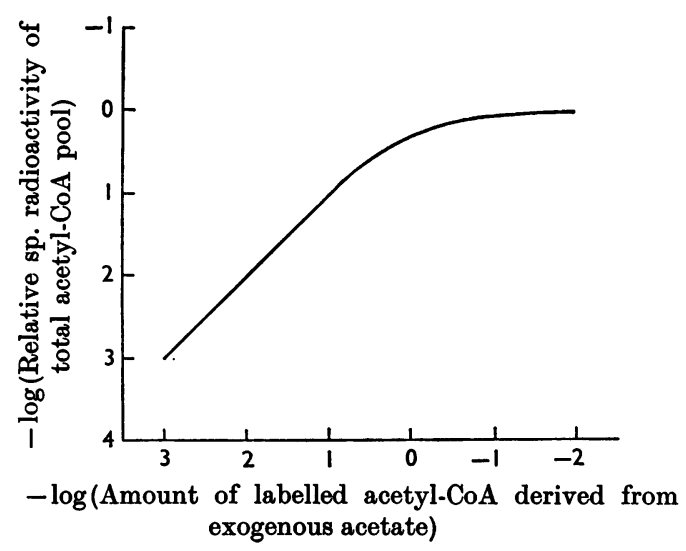

Fig. 2. Theoretical curve showing the effect of increasing conversion of labelled acetate into labelled acetyl-CoA on the relative specific radioactivity of the total acetyl-CoA pool. The latter is the specific radioactivity of that pool expressed as a fraction of the specific radioactivity of the labelled acetate from which the labelled acetyl-CoA molecules are derived. It is equal to $a /(a+b)$, where $a$ is the amount of labelled acetyl-CoA derived from exogenous acetate and $b$ is the amount of endogenously produced acetyl-CoA. The values for the relative specific radioactivity plotted above were calculated on the assumption that the endogenous acetyl-CoA pool is constant at unit amount $[-\log ($ amount $)=0]$.

the specific radioactivity of the total acetyl-CoA cease to increase linearly, and ultimately it reaches a plateau value equal to that of the labelled acetate. If it is assumed that labelled acetyl-CoA production is proportional to the concentration of the labelled acetate, and that the specific radioactivity of the tissue fatty acids is proportional to that of the total acetyl-CoA pool, then it can be seen that the curve of Fig. 2 has the same parameters as those of Fig. 1 and a similar shape. If this effect is dominant then the non-linear part of the relationship shown in Fig. 1 would indicate a deviation from tracer conditions since the concentration of endogenous acetylCoA, the first reactant in the chain, is being grossly altered by the 'tracer' acetate. This increased concentration might also produce secondary effects superimposed on the inverse dilution effect. Thus the conversion of acetyl-CoA into fatty acids might be relatively decreased, for instance by impaired malonyl-CoA production due to competition for ATP by acetyl-CoA synthetase, or by some other mechanism resulting in the channelling of a larger proportion of acetyl-CoA through metabolic pathways other than fatty acid synthesis.

Secondly, a similarity can be seen between the shape of Fig. 1 and the Michaelis-Menten relationship between enzymic reaction rate and substrate concentration. The analogy between the initial rate 
of a single enzymic reaction and the integrated incorporation of acetate into fatty acids must obviously not be taken too far, but the results could indicate that at high labelled-acetate concentrations the production of labelled acetyl-CoA is limited by the activity of acetyl-CoA synthetase or some other factor. If the resulting concentration of labelled acetyl-CoA is still low compared with that of the endogenous acetyl-CoA then no interference with the latter would occur, and in spite of the deviation of the response curve the higher concentrations of labelled acetate would not be infringing tracer conditions.

These mechanisms, which are mutually exclusive, are not the only ones that could be postulated as causing or contributing to the characteristic shape of the response curves, and the results of the present experiments do not provide evidence as to their occurrence or relative importance. It is thus possible that tracer conditions may obtain even on the non-linear part of the response curves (as discussed above for the second mechanism), but for one to be certain of operating under tracer conditions it is evident that the concentration of labelled acetate one uses must be limited to the range corresponding to the linear part of the response curves. In much published work this has not been done and lipogenesis in adipose tissue has been investigated with labelled acetate at concentrations as high as $60 \mathrm{~mm}$. Under these conditions the added labelled acetate can no longer be regarded as a tracer for endogenous acetyl-CoA, but as a substrate in its own right. The latter may be a legitimate use, but it must be distinguished from use under true tracer conditions.

As well as choice of an appropriate concentration for the added tracer, maintenance of this concentration during the experiment must be ensured. The results shown in Fig. 3 indicate that at tracer concentrations $(10 \mu \mathrm{M}$ and below) a significant proportion of the added labelled acetate may be metabolized, but that at higher concentrations, where the relative incorporation of acetate into fatty acids is less (see Fig. 1), the percentage utilization of the tracer is decreased. In the experiment shown in Fig. 3 the mean concentration of acetate in the medium during the incubation is probably not less than $80 \%$ of the initial value, even for the lower concentrations, so that the shape of the response curves (Fig. 1) will be little affected by utilization of tracer. In other experimental systems, however, this may not be so, and each case should be checked for this effect. A simple solution to the problem is to incubate the usual weight of tissue in medium containing the labelled material at the required low tracer concentration but of much greater volume than is customary, so that a sufficient reserve of tracer is maintained. The results of

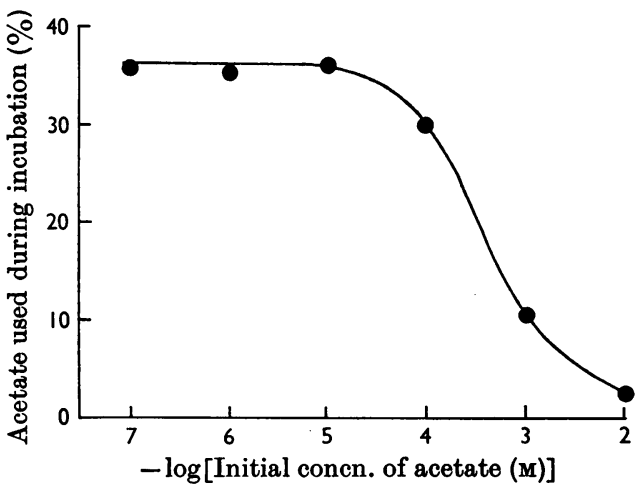

Fig. 3. Effect of initial acetate concentration on acetate utilization by rat adipose tissue in vitro. Incubation conditions were described in the Experimental section. The medium contained glucose (10 mM).

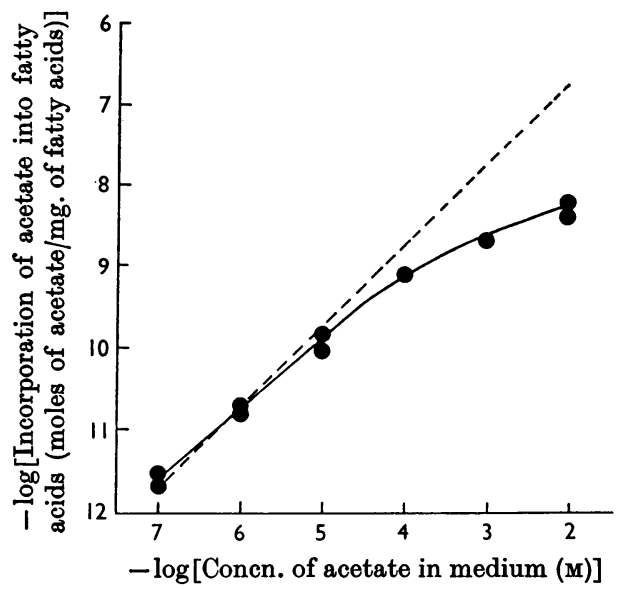

Fig. 4. Effect of concentration of labelled acetate in a large volume of medium on its incorporation into total fatty acids of rat adipose tissue in vitro. Experimental details were as for Fig. 1, except that each incubation vessel contained $50 \mathrm{ml}$. of medium with glucose at $10 \mathrm{mM}$. The broken line is the theoretical curve, as in Fig. 1.

such an experiment are shown in Fig. 4, in which $90 \%$ or more of the labelled acetate remained unused at the end of the incubation period, even at low initial acetate concentrations. This curve is very similar to those of Fig. 1 and confirms the general validity of the conclusions drawn from those curves for the particular experimental system being used.

It is noteworthy that the pattern of response shown in Fig. 1 was similar for widely differing absolute rates of incorporation of acetate into fatty acids. This incorporation is known to be stimulated 
in rat adipose tissue by glucose (e.g. Masoro, 1962) and in the present experiments the increase amounted to some 40-fold (compare the two curves of Fig. $1 a)$; yet the valid tracer range of concentration for labelled acetate (linear part of curve) was the same in the presence of glucose as in its absence. This implies that for any particular concentration of labelled acetate in the medium the addition of glucose did not affect the relative labelling of the acetyl-CoA pool used for fatty acid synthesis, i.e. the ratio acetyl-CoA derived from labelled acetate/ endogenous acetyl-CoA. If an increase in the rate of production of endogenous acetyl-CoA occurs as a result of glucose catabolism this must therefore be accompanied by an equal increase in the rate of conversion of labelled acetate into labelled acetylCoA; this could occur, for instance, by glycolysis providing extra ATP for increased acetyl-CoA synthetase activity. Such an increased rate of production of endogenous acetyl-CoA would not necessarily cause an increase in the overall tissue pool of acetyl-CoA. The utilization of acetyl-CoA, at least for fatty acid synthesis, is enormously increased in the presence of glucose, and the increased turnover of the pool might in fact lead to a diminution of its size. Of possible relevance to this is the observation that the concentration of acetylCoA in rat liver is raised under conditions of depressed fat synthesis (starvation) and lowered when fat synthesis is stimulated by carbohydrate feeding (Tubbs \& Garland, 1964).

Another possibility is that the acetyl-CoA derived from labelled acetate is not in equilibrium with that derived from glucose. The latter acetyl-CoA arises in the mitochondria (Sanadi, 1963) and is probably transferred to the site of de novo fatty acid synthesis by the pathway: intramitochondrial acetyl-CoA $\rightarrow$ intramitochondrial citrate $\rightarrow$ extramitochondrial citrate $\rightarrow$ extramitochondrial acetyl-CoA (Lowenstein, 1963). Acetyl-CoA synthetase on the other hand occurs extramitochondrially (de Duve, Wattiaux \& Baudhuin, 1962), and it is conceivable that labelled fatty acids may be synthesized from a labelled acetyl-CoA pool whose concentration and specific radioactivity are not greatly affected by changes in the concentration of acetyl-CoA derived from glucose.

The technical assistance of Mr I. C. Collie and Mr T. J. Rising is gratefully acknowledged.

\section{REFERENCES}

Balmain, J. H., Folley, S. J. \& Glascock, R. F. (1954). Biochem. J. 56, 234.

Braun, D. \& Geenen, H. (1962). J. Chromat. 7, 56.

Bray, G. A. (1960). Analyt. Biochem. 1, 279.

de Duve, G., Wattiaux, R. \& Baudhuin, P. (1962). Advanc. Enzymol. 24, 291.

Duncombe, W. G. \& Glascock, R. F. (1956). Biochem. J. $63,332$.

Emerson, R. J. \& Van Bruggen, J. T. (1958). Arch. Biochem. Biophys. 77, 467.

Favarger, P. (1965). In Handbook of Physiology, Section 5: Adipose Tissue, p. 19. Ed. by Renold, A. E. \& Cahill, G. F. Washington, D.C.: American Physiological Society.

Krebs, H. A. \& Henseleit, K. (1932). Hoppe-Seyl. Z. 210, 33.

Lowenstein, J. M. (1963). Symp. biochem. Soc. 24, 57.

Masoro, E. J. (1962). J. Lipid Res. 3, 149.

Sanadi, D. R. (1963). In The Enzymes, vol. 7, p. 307. Ed. by Boyer, P. D., Lardy, H. \& Myrbäck, K. New York: Academic Press Inc.

Tubbs, P. K. \& Garland, P. B. (1964). Biochem. J. 93, 550. 